\title{
Marie von Ebner-Eschenbach und der Naturalismus
}

Marie von Ebner-Eschenbach et le naturalisme

Marie von Ebner-Eschenbach and naturalism

\section{Ulrike Tanzer}

\section{(2) OpenEdition}

\section{Journals}

Édition électronique

URL : http://journals.openedition.org/austriaca/505

DOI : 10.4000 /austriaca.505

ISSN : 2729-0603

\section{Éditeur}

Presses universitaires de Rouen et du Havre

\section{Édition imprimée}

Date de publication : 1 juin 2018

Pagination : $39-53$

ISBN : 979-10-240-1233-9

ISSN : 0396-4590

\section{Référence électronique}

Ulrike Tanzer, „Marie von Ebner-Eschenbach und der Naturalismus“, Austriaca [Online], 86 | 2018,

Online erschienen am: 01 Juli 2020, abgerufen am 28 Januar 2021. URL: http://

journals.openedition.org/austriaca/505 ; DOI: https://doi.org/10.4000/austriaca.505 
Ulrike TANZER

Université d'Innsbruck

\section{Marie von Ebner-Eschenbach und der Naturalismus}

Marie von Ebner-Eschenbach stand dem Naturalismus distanziert, ja ablehnend gegenüber. Dies ist in der Forschung weitgehend common sense ${ }^{1}$ und lässt sich durch zahlreiche Äußerungen der Schriftstellerin belegen. In Ebner-Eschenbachs CEuvre finden sich zwar keine geschlossenen programmatischen Abhandlungen ${ }^{2}$. Dies gilt auch für ästhetische Fragen, die allerdings in den Erzählungen, Aphorismen, Korrespondenzen, Tagebuch- und Notizbuchaufzeichnungen immer wieder - unsystematisch und unterschiedlich gewichtet - thematisiert werden. Darüber hinaus finden sich in den Tagebüchern und Briefen Einträge zur Lektüre und zu Theaterbesuchen, die Hinweise auf die Naturalismus-Rezeption geben. Ebner-Eschenbach setzte sich verhältnismäßig früh mit den Werken Émile Zolas und Henrik Ibsens auseinander. Der Briefwechsel mit der Lyrikerin und Übersetzerin Josephine von Knorr ${ }^{3}$, der gerade für die $1850 e r$ und $1860 e r$ Jahre detailliert Auskunft über die Lese- und Schreibprojekte der beiden Freundinnen gibt, nimmt auch Bezug auf Ebner-Eschenbachs Zola-Lektüre. Knorr lebte ab 1876 zeitweilig in Paris und versuchte Anschluss an berühmte Künstlerinnen und Künstler zu finden. Besonders intensiv

1. Vgl. Christine Anton, „Marie von Ebner-Eschenbach und die Realismusdebatte. Schreiben als Auseinandersetzung mit den Kunstansichten ihrer Zeit", in Modern Austrian Literature 33/1 (2000), S. 1-15.

2. Vgl. Karlheinz Rossbacher, „Marie von Ebner-Eschenbach. Zum Verhältnis von Literatur und Sozialgeschichte, am Beispiel von Krambambuli“, in Österreich in Geschichte und Literatur 24 (1980), H. 2, S. 87-106, hier: S. 88.

3. Marie von Ebner-Eschenbach - Josephine von Knorr, Briefwechsel 1851-1908. Kritische und kommentierte Ausgabe. Mit Marie von Ebner-Eschenbachs historischer Studie Carl I. von England und die hervorragenden Charactere seiner Zeit (1854), hg. v. Ulrike Tanzer, Irene Fußl, Lina Maria Zangerl und Gabriele Radecke. 2 Bde., Berlin/Boston, De Gruyter, 2016. 
ist die Beziehung zur Schriftstellerin Louise-Victorine Ackermann, zum Schriftsteller Iwan Turgenew und zum Journalisten Alfred Marchand, der als Redakteur der Zeitschrift Temps die österreichische Literatur in Frankreich fördert. Am 13. Februar 1880 schreibt Ebner-Eschenbach an die in Paris weilende Freundin: „Liebe Sephine ich bitte Dich schick mir gleich - gleich eine Photographie von Zola. Ich habe l'Assommoir gelesen und möchte gern wissen wie der Mensch aussieht der etwas so Ungeheures schreiben kann. " ${ }^{4}$ Wenige Tage später folgt Josephine von Knorrs Brief aus Paris. Darin zeigt sich Knorrs Vorliebe für das Sammeln von Fotografien, eine Leidenschaft, die sie mit Persönlichkeiten wie Johann Wolfgang von Goethe oder auch der österreichischen Kaiserin Elisabeth teilt:

Zola findest $\mathrm{Du}$ als Beilage. Ich kenne nun par photographie die hervorragendsten der geistigen Miliz Frankreichs. Den hübschesten finde ich Paul de Cassagne, das Napoleonische, was glaube ich Sardou haben soll, finde ich nicht heraus; Alexandre Dumas fils ist sehr alt geworden und sieht mehr wie ein père aus; Daudet ist hübsch sieht aber auf der Photographie doch aus wie ein homme-chien; Cittré ist schon mehr Mumie; Sir Hiacynthe ist ein Karakterkopf; von Mme Adam konnte ich keine Photographie bekommen sie interessirt mich, scheint unter den Französinnen die erste politische Rolle zu spielen; soll sehr schön sein, was ihr dazu helfen mag. [... $]^{5}$.

Ebner-Eschenbach antwortet mit „Dank für die Photogr: dreimal unterstrichenen Dank! Sie ist im höchsten Grade charakteristisch. Die Nase aufgespannt wie ein Regenschirm - da kann man freilich so manches riechen. [... $]^{\text {“6 }}$ Der Erhalt der Sendung wird auch im Tagebuch festgehalten:

Sephine schickt die Photographie Zolas. Höchst charakteristisch, das Gesicht eines französischen Arbeiters breite Stirn die Haare vorn schon ein wenig dünn, zu Berg stehend breite Augenbrauen, ruhige, harte, verächtlich blickende Augen, das linke viel größer als das rechte, eine Nase aufgespannt wie ein Regenschirm (da kann man freilich manches riechen) dichter Schnurbart

4. Marie von Ebner-Eschenbach an Josephine von Knorr, Wien, 13.2.1880, in Marie von Ebner-Eschenbach - Josephine von Knorr, Briefwechsel 1851-1908, Bd. 1, 2016, S. 443 .

5. Josephine von Knorr an Marie von Ebner-Eschenbach, Paris, 19.2.1880, in: Marie von Ebner-Eschenbach - Josephine von Knorr, Briefwechsel 1851-1908, Bd. 1, 2016, S. $444 f$.

6. Marie von Ebner-Eschenbach an Josephine von Knorr, Wien, 23.2.188o, in: Marie von Ebner-Eschenbach - Josephine von Knorr, Briefwechsel 1851-1908, Bd. 1, 2016, S. 445 . 
der den Mund nicht verdeckt, vorgeschobene Unterlippe, der Mund schief, kurzer Vollbart kurzer Hals breite Schultern ${ }^{7}$.

Ebner-Eschenbachs kritischer Blick auf Zola sollte sich nicht mehr ändern. Einige Jahre später findet sich ein weiterer Tagebuch-Eintrag: „Abends allein wir lasen Zola's Germinal zu Ende. Der allerletzte Schluß sehr schön anderthalb Kapitel vorher unerlaubt, weil überflüßig grauenhaft. “8 Auch Josephine von Knorr stand den (französischen) Naturalisten ablehnend gegenüber. Sie war - wie die Dichterinnen Auguste Hyrtl, Marie von Najmajer und Marie Eugenie delle Grazie Mitglied der Gesellschaft Iduna, eines konservativen Kreises von Wiener Schriftsteller/inne/n (1891-1904), der sich gegen den naturalistischen Einfluss in der Literatur der Jahrhundertwende wandte. Interessant ist in diesem Zusammenhang Marie Eugenie delle Grazie (1864-1931), die mit dem Bergarbeiterdrama Schlagende Wetter (UA: 1900) „das einzige naturalistische Drama der österreichischen Literatur" ${ }^{\text {" } 9}$ schrieb, sich dann aber dem Katholizismus zuwandte. Damit verlor sie alle ihre bisherigen Publikationsorte und wurde kaum mehr wahrgenommen. 1916 erhielt sie den Ebner-Eschenbach-Preis für ihr Werk. 1919 erschien ihr Antikriegsroman Homo ... Der Roman einer Zeit ${ }^{10}$.

Die oben angeführte Beschreibung Zolas ist aber auch in einem anderen Zusammenhang von Interesse: Sie steht in der Tradition der Physiognomik Lavaters, dessen Methodik in Charles Darwins Abhandlung Der Ausdruck der Gemüthsbewegungen bei den Menschen und den Tieren (1872) wiederaufgegriffen und weitergeführt wurde ${ }^{11}$. EbnerEschenbach, die schon früh naturwissenschaftliche und philosophische Studien betrieb, las das Buch - ebenso wie Minna Kautsky und Hugo von Hofmannsthal - mit großem Interesse. In ihr Tagebuch notierte sie: „Darwin Der Ausdruck etc. S. 39. ,Nach einem einmaligen heftigen Scheuwerden, wenn das Pferd erregt ist u. das Blut reichlich durch das Gehirn fließt, ist es sehr geneigt, von Neuem zusammenzufahren;

7. Marie von Ebner-Eschenbach, Tagebücher III. 1879-1889. Kritische Texte und Deutungen. Hg. von Karl Konrad Polheim und Norbert Gabriel unter Mitwirkung von Markus Jagsch und Claus Pias, Tübingen, Niemeyer, 1993, 22.2.1880, S. 15.

8. Marie von Ebner-Eschenbach, Tagebücher III, 27.3.1886, S. 568.

9. Evelyne Polt-Heinzl, Ringstraßenzeit und Wiener Moderne. Porträt einer literarischen Epoche des Übergangs, Wien, Sonderzahl, 2015, S. 8.

10. Ebd., S. 41.

11. Werner Michler, Darwinismus und Literatur. Naturwissenschaftliche und literarische Intelligenz in Österreich, 1859-1914. Wien, Köln, Weimar (= Literaturgeschichte in Studien und Quellen, Bd. 2), Böhlau, 1999, S. 223. 
dasselbe gilt für kleine Kinder.“12 Und wenige Tage später: „Fritzels erste Schreibversuche. Darwin hätte sich gefreut üb. die Bewegungen die das Kind mit den Händen machte, während ich vorschrieb. “13 $\mathrm{Zu}$ sammenfassend hält Ebner-Eschenbach in ihrem Tagebuch fest: „Der hat seinen Darwin schlecht gelesen, der nicht weiss welch ein inniger Zusammenhang zwischen dem Herzen u. dem Gehirn besteht." ${ }^{14}$

Der norwegische Schriftsteller Henrik Ibsen wiederum polarisierte. Im April 1891 fand die Wiener Ibsen-Woche statt. Ibsen wohnte auf Einladung des neuen Burgtheaterdirektors Max Burckhard der Wiener Erstaufführung der Kronprätendenten bei, die vom Publikum stürmisch gefeiert wurde. Die Herausgeber der Modernen Rundschau veranstalteten am 11. April 1891 ein Festbankett im Hotel Kaiserhof, die Festrede hielt der Wiener Germanist Jakob Minor. Am 8. September 1881 war Ibsens Nora am Wiener Stadttheater aufgeführt worden, knapp zwei Jahre nach der Uraufführung im Dezember 1879 in Kopenhagen. Zu beiden Anlässen finden sich keine Eintragungen in Ebner-Eschenbachs Tagebuch. Ende Jänner 1885 notiert sie nach dem Besuch einer Vorlesung von Georg Brandes im Wiener Ehrbar-Saal: „Brandes sagte, daß die deutsche Literatur zu viel Rücksicht auf das Publikum nehme statt auf das Publikum zu wirken lasse sie sich vom Publikum beeinflußen u. herunter zerren. Anders im Norden. Man weiß wenn ein neuer Roman v. Ibsen erscheint, der wird von uns das schlimmste aussagen aber man verschlingt ihn. “15 Am 22. Februar 1889 sieht Marie von EbnerEschenbach Ibsens Frau am Meer: „Mir ganz unverständlich, Moriz ergriff die Flucht. Wie gern hätte ich dasselbe gethan. " ${ }^{16}$ Und in ihr Arbeitsheft notiert sie: „Im tiefsten Herzen Ibsens sitzt etwas Widerwärtiges, ein grauer, harter, kantiger Eiszapfen." ${ }^{17}$

So skeptisch Marie von Ebner-Eschenbach dem Naturalismus gegenüber stand, so wenig konnte sie auch der Bewegung Jung-Wien abgewinnen. Sie geht mit deren Werken hart ins Gericht. So heißt es etwa über Arthur Schnitzlers Roman Der Weg ins Freie von 1908: „Was wird in dem Buch geschwatzt! Welche unerträgliche Selbstbespiegelung!

12. Marie von Ebner-Eschenbach, Tagebücher III, 2. Juni 1882, S. 222.

13. Marie von Ebner-Eschenbach, Tagebücher III, 12. Juni 1882, S. 225 f.

14. Marie von Ebner-Eschenbach, Tagebücher III, Anhang 1882, S. 285.

15. Marie von Ebner-Eschenbach, Tagebücher III, 30.1.1885, S. $466 f$.

16. Marie von Ebner-Eschenbach, Tagebücher III, 22.2.1889, S. 737.

17. Marie von Ebner-Eschenbach, Arbeitsheft mit Parabeln und Zitaten. I.N. Ia 79.194, undat., Wienbibliothek im Rathaus. 
Der Held ist ja Schnitzler selbst. "18 Bei der Beurteilung bedient sie sich der „gesund/krank“-Dichotomie:

Hysterische Männer Aestheten, verdrehte sogenannte Poeten u. Künstler haben wir gerade genug die laufen auf allen Gassen herum, sammeln in den Kaffeehäusern lungern in den Salons herum. Wen wir brauchen sind einfach an Leib u Seele gerade gewachsene, natürliche Leute Männer u. Frauen [...] wie jedes große angeborene Talent gesund schlicht u natürlich die einen Ekel haben vor den ungesunden lüsternen frechen. ${ }^{19}$

Dies bleibt nicht die einzige Eintragung, in der sie die Werke der Modernen als „krank“ bezeichnet: „Sie lesen die Modernen? Ja wohl, man geht als Arzt doch nach Bombay um die Pest zu studiren. ${ }^{20}$ Ihr Schriftstellerkollege Ferdinand von Saar fühlt sich durch die wohlwollende, wenngleich nicht unkritische Anerkennung der jungen Autoren-Generation geehrt, kann aber mit deren Texten ebenso wenig anfangen. So heißt es etwa in einem Brief Saars an Nelly von Gomperz, der Nichte seiner Gönnerin Josephine von Wertheimstein, über Hofmannsthals dramatische Studie Gestern: „Das ,Gestern“ hatte mir wirklich kaum Eindruck gemacht - aber auf Andere. Daher kann ja wirklich nur die Schuld an mir liegen. Dennoch können ein paar abgerissene Szenen keinen Anspruch auf wirklichen Werth erheben, selbst wenn es die genialsten und großartigsten wären.“" ${ }^{21}$ Saar sah sich als „ein Übergang“, es gehe „eben nicht auf einen Schlag. Aber ich bin doch der Übergang. "22 Insgesamt sah er aber seine Generation als zu wenig eigenständig wahrgenommen. 1905 schreibt Saar an Ebner-Eschenbach, es sollte einmal „ein österreichischer Litterarhistoriker die Periode nach Grillparzer in Angriff nehmen und M. von Ebner-Eschenbach, Hamerling, Anzengruber, Rosegger, Saar vereint behandeln und die Zusammenhänge nachweisen,

18. Marie von Ebner-Eschenbach, Tagebücher VI. 1906-1916. Kritische Texte und Deutungen. Hg. von Karl Konrad Polheim und Norbert Gabriel. Tübingen, Niemeyer, 1997, Anhang 1909.

19. Marie von Ebner-Eschenbach, Notizbuch, I.N. Ia 81.180, um 1911, Wienbibliothek im Rathaus.

20. Marie von Ebner-Eschenbach, Notizbuch, I.N. Ia 81.188, undat., Wienbibliothek im Rathaus.

21. Ferdinand von Saar an Nelly von Gomperz, Brief v. 7.5.1892, I.N. 122.171 (Wienbibliothek im Rathaus, Wien). Vgl. auch Hugo von Hofmannsthal, Briefwechsel mit Marie von Gomperz 1892-1916. Mit Briefen von Nelly von Gomperz, hg. von Ulrike Tanzer. Freiburg i.Br. (= Rombach Wissenschaften), Rombach, 2001.

22. Hermann Bahr, Tagebücher, Skizzenbücher, Notizhefte. Bd. 2: 1890-1900. Hg. von Moritz Csáky. Wien, Köln, Weimar, Böhlau, 1996, S. 128. 
die trotz der Verschiedenheit der dichterischen Individualitäten, zwischen den Genannten bestehen. “23

Als Marie von Ebner-Eschenbach anlässlich ihres 70. Geburtstags am 13. September 1900 als erste Frau das Ehrendoktorat der Universität Wien erhält, so wird ihr diese Würde auf Vorschlag von Professor Jakob Minor zuerkannt. In seiner Rede macht Minor, Ordinarius für deutsche Sprache und Literatur in der Nachfolge Erich Schmidts, deutlich, dass Ebner-Eschenbach auch als eine „Symbolgestalt“ ${ }^{24}$ des Ausgleichs preiswürdig erscheint. Ihr Herz sei „von Kindheit auf zwischen den Slaven und den Deutschen“ geteilt, und dabei weise "diese deutsche und zugleich wieder echt österreichische Dichterin“ doch „über die nationalen Gegensätze hinaus auf die höhere Menschenliebe“. Marie von EbnerEschenbach sei den Werten der Klassik verbunden und zugleich „ein echtes und rechtes Kind der neuen Zeit“. Sie stehe in der Mitte „Zwischen der alten und der neuen Schule“, zwischen dem Idealismus und dem Realismus: „sie kann den Ruhm für sich in Anspruch nehmen, nach dem Tode Fontane's der einzige Schriftsteller der älteren Generation zu sein, der sich bei Alt und Jung der gleichen Anerkennung erfreut. ${ }^{\text {" } 5}$ Festgehalten wird das von Anton Bettelheim mitgestaltete Bild einer Dichterin der Güte und des Mitleids, gepriesen werden angebliche weibliche Tugenden und Gefühle. Die junge Schriftstellergeneration reihte sich wie selbstverständlich in die Schar der Gratulanten ein. EbnerEschenbach selbst sah mit zunehmendem Alter ihre Stellung im literarischen Feld illusionslos-nüchtern: „Ich komme mir manchmal wie der Papagei [vor] den niemand mehr verstand. Er war so alt, daß er eine tote Sprache sprach. ${ }^{26}$

23. Ferdinand von Saar an Marie von Ebner-Eschenbach, Brief v. 20.1.1905, I.N. 49972 (Wienbibliothek im Rathaus, Wien). Trotz umfangreicher Untersuchungen, besonders Karlheinz Rossbachers Studie zu Literatur und Liberalismus, hat die österreichische Literatur der zweiten Hälfte des 19. Jahrhunderts nach wie vor einen schweren Stand.

24. Daniela Strigl, Berühmt sein ist nichts. Marie von Ebner-Eschenbach. Eine Biographie. Salzburg, Wien, Residenz, 2016, S. 361.

25. Zit. nach Anton Bettelheim, Marie von Ebner-Eschenbach. Biographische Blätter. Berlin, Paetel, 1900, S. 237, $246 f$.

26. Marie von Ebner-Eschenbach, Notizbuch mit Aphorismen, I.N. Ia 79.160, undat., Wienbibliothek im Rathaus. 
Das Problem der Vererbung - eines der wichtigsten Themen des Naturalismus - findet sich in Ebner-Eschenbachs Oeuvre immer wieder. Der Roman Das Schädliche etwa erzählt in Form einer Lebensbeichte die Geschichte einer unglücklichen Liebe des Erzählers zu Edith. Der Text erschien 1894 in Heft 36 und 37 von Die Romanwelt. Zeitschrift für die erzählende Litteratur aller Völker, herausgegeben von Otto NeumannHöfer und Felix Heinemann. Der Publikationsort ist bemerkenswert, zählte doch die Romanzeitschrift zu den Periodika, die von Anfang an der Moderne gegenüber aufgeschlossen waren. ${ }^{27}$ Die Geschichte des Adeligen Franz beginnt bei seiner Geburt im Jahre 1829 und reicht bis zu seiner Ehe und seiner Vaterschaft. Nach dem Tod seines Vaters, der stirbt, ohne sich mit seiner Frau zu versöhnen, lernt Franz Edith kennen, die mittlere von drei Töchtern einer wohlhabenden Industriellenfamilie. Sie ist die ungeliebte Tochter, doch mit großem künstlerischen Talent und geheimnisvoller Ausstrahlung. Ihre Begabung bedeutet ihr nichts, sie wendet all ihr Sinnen und Trachten Franz zu, der sie trotz der Bedenken seiner Mutter und ihrer Eltern heiratet. Kurz nach ihrer Heirat wird eine Tochter geboren, Eleonore, die sich zu einem Abbild Ediths entwickelt. Die Ehe ist von Ediths Eifersucht überschattet. Dabei ist sie der gesellschaftliche Mittelpunkt, umschwärmt von Männern und Frauen. Sie unterschlägt ein Telegramm, das sie von der Ballsaison in Wien zurück zur sterbenden Mutter ruft. Als das Ehepaar zu spät das Schloss erreicht und die Mutter schon tot ist, ist Franz „unheilbar verwundet ${ }^{\star 28}$ Der innere Bruch ist nicht mehr zu kitten. Nach verschiedenen Affären Ediths kommt es zur Scheidung. Das Kind Lore bleibt beim Vater zurück.

Lore wächst unter der Aufsicht der streng religiösen Schwester Ediths, Maud, heran. Lore ähnelt ihrer Mutter, ohne aber deren Liebesfähigkeit zu besitzen. Franz liebt seine Tochter abgöttisch und glaubt, dass das Schlechte nicht angeboren sei und deswegen ,ausgerottet" (Sch 610)

27. Polt-Heinzl, 2015, S. $172 \mathrm{f}$.

28. Marie von Ebner-Eschenbach, Das Schädliche, in: MvEE, Das Gemeindekind, Novellen, Aphorismen. München: Winkler 1956, 579-639, hier: S. 598. Zitiert nach der Sigle Sch. 
werden könne. Eine Szene, die auf den Titel der Erzählung anspielt, macht dies deutlich:

„Siehst du“, sagte ich, „nicht nur erlaubt ist's mir, ein so gefährliches Tier zu töten, ich muß das tun, um unser und der andern willen. Heute hat es unsre Hennen erwürgt, ihr Blut ausgetrunken und ihre Eier, morgen würde es beim Nachbarn einbrechen. Es ist gut und recht, das Schädliche wegzuschaffen aus der Welt."

„Das Schädliche?“ wiederholte sie. „Nennt man einen Marder das Schädliche?"

„Man nennt in der Jägersprache alle Tiere so, die sich vom Fraße nützlicher Tiere nähren, des guten, armen Federviehs im Haus, im Wald und auf dem Feld, des kleinen Hasen, der jungen Rehe." (Sch 613)

Ähnlich wie in Ibsens Drama Ein Volksfeind (1882) ${ }^{29}$ verwendet EbnerEschenbach einen Tiervergleich. Der Ich-Erzähler hatte in der Nacht:

einen furchtbaren Traum. Ich lag da, wehrlos und gelähmt an allen Gliedern, und sah einen Marder an mich heranschleichen, mit leisen, leichten Schritten. Es war ein unvergleichlich schönes Raubtier, ich konnt's nicht hassen, ich mußte es bewundern, während es mein Herzblut trank, denn es hatte Lores Augen. In Angstschweiß gebadet wacht ich auf ... (Sch 613)

Der Vater muss erkennen, dass weder strenge Erziehung noch Liebe zum Erfolg führen. Lore quält ihren Milchbruder Rupert, den Sohn eines Gutsbeamten, sie macht weitere Eroberungen, fühlt sich zu einem Künstler und Gelehrten hingezogen und plant gleichzeitig die Verlobung mit Fürst Nordhausen. Gerade im Vergleich mit Rupert lässt Ebner-Eschenbach die Frage der Vererbung offen. Während Rupert in keiner Weise nach seinen Eltern gerät, ist Lore ein ins Negative gewendetes Abbild ihrer Mutter. Beide werden als femmes fatales dargestellt, die in ihrer maßlosen Vitalität die (männliche) Ordnung gefährden.

Und dann wieder: Es gibt keine erbliche Belastung? - Possen! Edith wiederholte sich in jedem Blutstropfen ihres Kindes. Lores Fehler waren die Fehler ihrer Mutter, nur zur Potenz erhoben... Die Falschheit zum Beispiel. Das wenige Gute freilich fehlte. Lore war einer großen Liebe unfähig. Bei ihr schlich sich überall Berechnung ein. Wie sie so geworden? Nein, nicht geworden, sie war so geboren, hat sich ihren innersten Gesetzen gemäß entfaltet, wie es in ihrer urkräftigen Natur lag, allen äußeren Einwirkungen zum Trotze. - (Sch 624f.)

29. Henrik Ibsen, Gespenster. Ein Volksfeind. Neu übers. u. hg. v. H. Gimmler. Nördlingen 1989, S. 182. 
Ebner-Eschenbach beschreibt in dieser Erzählung, die ihr Kollege

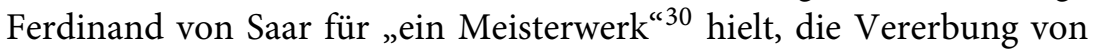
Charaktereigenschaften der Mutter auf die Tochter. Lore wird letztlich wie der tragische Held im Naturalismus, schuldlos schuldig'. In die Vererbung als determinierende Instanz ist nicht mehr einzugreifen. Die Erzählung steuert schließlich unausweichlich auf die Katastrophe zu. Der Vater verhindert durch sein kurzes Zögern im entscheidenden Moment den Mord Ruperts an Lore nicht.

Ich wollte aufschreien: „Zurück!“ aber das Wort erstarb mir im Munde. Alle Pein der Vergangenheit und der Gegenwart, alle Schauder vor der Zukunft ballten sich in eine Anklage zusammen. Sie lebt zum Unheil eines jeden, der ihr naht, ist das Schädliche; fort mit dem Schädlichen aus der Welt. Das Schicksal walte! Laß es geschehn! (Sch 637)

Lore fällt tödlich getroffen. Sie stirbt, ohne einen Funken von Reue gezeigt zu haben: „Ich sterbe - auch gut.“ (Sch 637) Rupert erschießt Lore von einer Position, wo er keinen „besseren Stand auf das Wild“ (Sch 636) hätte haben können. Mit dieser Metapher wird Lore zu einem Tier, wie schon zuvor im Gespräch über den Marder und das Schädliche. Ähnlich verfährt übrigens Ferdinand von Saar in seiner 1889 erschienenen Novelle Die Troglodytin, in der die sexualisierte Frau als „Katze“, zuvor positiver als „Reh“ und „Spottdrossel“ bezeichnet wird. Saars Protagonistin wird - ebenso wie Lore - als „erotische Abenteurerin und potentielle Ordnungsstörerin “31 eliminiert. Anders als Lore gehört Maruschka Kratochwil dem Subproletariat an. Das UnkontrolliertTriebhafte wird hier den Unterschichten zugeschrieben. In EbnerEschenbachs Erzählung bleibt der hin- und hergerissene Vater zurück, der sich selbst Vorwürfe macht, gleichzeitig aber in der Gewissheit lebt, die Familie vor einer Schmach bewahrt zu haben. Der Schluss bricht mit der Brieffiktion, das Ende wird aus traditioneller Erzählerperspektive erzählt:

In diesem Haß hat der Mann Rettung vor dem Zweifel gesucht, der ihn mit wachsender Qual bedrängt haben mag, während er seine traurige Geschichte niederschrieb. Als sein Freund sie gelesen hatte, eilte er zu ihm, fand ihn aber nicht mehr lebend. (Sch 639)

30. Ferdinand von Saar an Marie von Ebner-Eschenbach, 28.12.1894. In: Briefwechsel zwischen Ferdinand von Saar und Maria von Ebner-Eschenbach, hg. v. Heinz Kindermann. Wien, Wiener Bibliophile Gesellschaft, 1957, S. 107.

31. Karlheinz Rossbacher, Literatur und Liberalismus. Zur Kultur der Ringstraßenzeit. Wien, Jugend \& Volk, 1992, S. 332. 
Peter C. Pfeiffer hat in seiner Interpretation der Erzählung die Verbindung zu naturalistischen Themen nicht in den Vordergrund gestellt. Vielmehr sieht er bei Ebner-Eschenbach „die Frage nach der Vererbbarkeit von Charaktereigenschaften als ideologisch und geschlechtsspezifisch besetzt". Ein solches Denken, dies mache die Erzählung deutlich, könne auch als „Mittel der Kontrolle und Zerstörung gegen die Frauen eingesetzt werden". ${ }^{32}$ Dies unterscheidet den Text etwa von Ludwig Anzengrubers Drama Das vierte Gebot (UA: 1877), in dem - so der Wiener Kritiker und Schriftsteller Adam Müller-Guttenbrunn - die "Theorie von den Sünden der Väter" literarischen Ausdruck gefunden habe. Anzengruber dürfe sich „berühmen, als Dramatiker der erste gewesen zu sein, der sich zu jenem naturwissenschaftlichen Glauben bekannt, dessen Apostel Ibsen geworden ist. “33 Anzengrubers Drama scheint alle Charakteristika eines naturalistischen Stückes zu erfüllen. Der heruntergekommene Handwerkerstand wird ebenso schonungslos gezeigt wie die desaströsen Verhältnisse der Hausherrenfamilien. Die Tradition des Wiener Vorstadttheaters wird hier endgültig verabschiedet, ein didaktisch-aufklärerischer Impetus bleibt aber sichtbar.

Die „Fallgeschichte des Mördersohns Pavel Holub“34 schließlich ist ein eindrückliches Beispiel dafür, wie Marie von Ebner-Eschenbach sich im Verlauf der Erzählung von deterministischen Prämissen distanziert und die Grenzen des Möglichen auslotet. Gleich zu Beginn des Romans Das Gemeindekind (1887) werden die wichtigsten Koordinaten genannt: Schauplatz der Geschichte, die auf eine wahre Begebenheit zurückgeht, ist ein Dorf in der mährischen Provinz. Ausgangspunkt der Handlung

32. Peter C. Pfeiffer, Marie von Ebner-Eschenbach. Tragödie, Erzählung, Heimatfilm. Tübingen, Narr Francke, 2008, S. 123.

33. Adam Müller-Guttenbrunn, „,Gespenster'. Familiendrama von Henrik Ibsen. Im Wiener Deutschen Volkstheater zum erstenmal aufgeführt am 21. November $1890^{\circ}$. [Deutsche Zeitung (Wien), 25.11.189o], in: A. M.-G., Feuilletons. Erschienen in der Wiener „Deutschen Zeitung“ 1886 bis 1892 . Bearb. u. eingel. v. N. Britz. Tl 2 (1889 bis 1892). Wien, 1978, S. 484-494, S. 484.

34. Philipp Hubmann, „,ich, dem's alle Tage geschehen kann, er weiß nicht wie, daß er Einen erschlagen muß'. Biopolitik und Risikobewusstsein in Marie von EbnerEschenbachs Das Gemeindekind“, in Thomas Wegmann und Martina King (Hg.), Fallgeschichte(n) als Narrativ zwischen Literatur und Wissen. Innsbruck (= Germanistische Reihe 84), innsbruck university press, 2016, S. 167-193, hier S. 167. 
ist der Prozess gegen den Ziegelschläger Martin Holub und dessen Frau Barbara im Oktober 1860. Der Mann wird für den Raubmord an einem Pfarrer hingerichtet, die Frau, die jede Aussage verweigert, wegen Beihilfe zu zehn Jahren Zuchthaus verurteilt. Die beiden Kinder, der dreizehnjährige Pavel und seine zehnjährige Schwester Milada, werden der Gemeinde als Pfleglinge übergeben. Während das Mädchen in die Obhut der Baronin und Ortspatronin kommt, wird ihr Bruder der verrufenen Familie des Dorfhirten Virgil am Rande des Dorfes überantwortet. Mit der Rückkehr der Mutter aus dem Gefängnis im Jahre 1870 endet der Roman. Die Wahl dieses Zeitraums ist von Bedeutung: Nach dem Ende der Feudalherrschaft zählte zu den Aufgaben der Gemeinde auch das Polizei- und Fürsorgewesen, dem oft mehr schlecht als recht nachgekommen wurde. Der Einfluss des Adels war zwar zurückgedrängt, realpolitisch aber nicht beseitigt. Dass sich die Baronin Miladas annimmt und sie in einem Kloster unterbringt, ist private Fürsorge, keine Verpflichtung. Die Dekade zwischen 1860 und 1870 deckt sich zudem mit Aufstieg und Blütezeit des Liberalismus. Die Konflikte zwischen den Nationalitäten verschärfen sich. Die Auseinandersetzungen zwischen Staat und Kirche münden in der formellen Kündigung des Konkordats im Sommer 1870. Die soziale Frage bleibt weitgehend unbeantwortet. Die Geschichte des Gemeindekindes Pavel, dies machen die wenigen zeitgeschichtlichen Anmerkungen deutlich, geht über die Schilderung eines individuellen Schicksals hinaus. Marie von Ebner-Eschenbach verlegt ihre Beobachtung zeitspezifischer Konflikte fern der Metropole an den Rand des Reiches, in den „Umkreis einer noch dominant feudal patriarchalischen Schloss- und ethnischen Misch-Gesellschaft“ ${ }^{\star 35}$. Mit dem Motto „Tout est l'histoire“ aus George Sands Autobiographie Histoire de ma vie (1854) wird dies nochmals unterstrichen. Karlheinz Rossbacher hat etwa auf die Verbindungen zwischen dem ländlichen Mikrokosmos und dem Makrokosmos der Habsburgermonarchie am Beispiel der Vorurteile und des Wahns der Dorfbewohner aufmerksam gemacht: „Die Struktur des Vorurteils als Wahn und Aberglaube bei den Dörflern ist dieselbe wie die des Nationalismus als Wahn und

35. Hugo Aust, „Am Rande des Realismus: Marie von Ebner-Eschenbachs späte Ehegeschichten aus Dorf und Schloss", in Claudia Meyer (Hg.), Bis zum Lorbeer versteig ich mich nicht. Festschrift für Jürgen Hein. Münster, Ardey-Verlag, 2007, S. 225-235, hier: S. 225. 
Vorurteil zwischen den Völkern. Auch deshalb also ist alles, was im Roman geschieht, Geschichte. “36

Die Konzentration auf das Dorf erlaubt eine präzise Beschreibung der gesellschaftlichen Schichten, von der kirchlichen Hierarchie über die liberalen Honoratioren bis zu den Bauern. Pavel gegenüber versagen die meisten: die Gebildeten, die Institution der Gemeinde, die Kirche, das "Schloss" und „der" Liberalismus. Nur wenige zeigen sich als Lebenshelfer, vor allem der Lehrer Habrecht und der Schmied Anton.

[...] er war das verkörperte Elend, der Bub! - Nicht durch die Schuld der Natur. Sie hatte es gut mit ihm gemeint und ihn kräftig und gesund angelegt; das zeigte die breite Brust, das zeigten die roten Lippen, die starken, gelblich schimmernden Zähne. Aber die wohlwollenden Absichten der Natur waren zuschanden gemacht worden durch harte Arbeit, schlechte Nahrung, durch Verwahrlosung jeder Art. Wie der Junge dastand mit dem wilden braunen Haargestrüpp, das den stets gesenkten Kopf unverhältnismäßig groß erscheinen ließ, mit den eingefallenen Wangen, den vortretenden Backenknochen, die magere derbe Gestalt von einem mit Löchern besäten Rock aus grünem Sommerstoff umhangen, die Füße mit Fetzen umwickelt, bot er einen Anblick, abstoßend und furchtbar traurig zugleich, weil das Bewußtsein seines kläglichen Zustandes ihm nicht ganz verlorengegangen schien. ${ }^{37}$

Mit diesen Worten wird Pavel eingeführt. Er wird dem Gemeindehirten übergeben, der mit seiner Familie ,ein Stübchen in der vorletzten Kaluppe am Ende des Dorfes" (GK 138) bewohnt. Damit ist nun nicht bloß die geographische Lage angegeben, sondern auch der soziale Rang der „Verrufensten des Ortes“(GK 138). Ebner-Eschenbach lehnt es ironisch ab, sich bei der Schilderung der Hütte des „Pinsels eines Realisten“" (GK 138) zu bedienen, womit im zeitgenössischen Sprachgebrauch die Naturalisten gemeint sind. Mit diesem knappen Hinweis erteilt die Autorin der Theorie von der Milieudeterminiertheit des Menschen eine Absage. Vielmehr zeigt sie gerade am Beispiel Pavels, der zunächst am äußersten Rand der Gesellschaft positioniert ist, die Möglichkeiten auf, trotz aller Rückschläge dem eigenen Milieu zu entfliehen. Sein vorbestimmtes Schicksal zu überwinden gelingt Pavel durch Charakterstärke,

36. Rossbacher, Literatur und Liberalismus, 1992, S. 264 f.

37. Marie von Ebner-Eschenbach, Das Gemeindekind., in MvEE, Aus Franzensbad. Das Gemeindekind, hg. v. Evelyne Polt-Heinzl und Ulrike Tanzer. Vorwort von Ulrike Tanzer. Mitarbeit von Lina Maria Zangerl. St. Pölten, Salzburg, Wien (= Leseausgabe in vier Bänden, Bd. 1), Residenz, 2014, S. 127-347, hier: S. 146f. - Im Folgenden zitiert mit der Sigle GK. 
Selbstverantwortung und Eigeninitiative. Sein Verständnis für Technik - am Beispiel der Episode mit der Dampfmaschine - erweist sich als förderlich für sein Selbstbewusstsein und damit als zukunftsträchtig. Entscheidend ist aber auch die verständnisvolle Hilfe von außen, die nicht von Ideologien oder Institutionen kommt, sondern aus zwischenmenschlichen Bindungen erwächst.

Pavels Ich-Profilierung wird vor allem als Weg aus der Sprachlosigkeit dargestellt. Gleich am Beginn wird geschildert, dass Mutter und Sohn „rastlos, finster und stumm“ (GK 130) rackern. Die Sätze, die Pavel von sich gibt, sind kurz und abgehackt - zunächst an seine Schwester gerichtet („Sag, daß du hungrig bist!“), dann Trotzreaktionen gegen die anderen („Weißt schon.“). Mit wenigen Strichen wird hier die Situation eines traumatisierten, allein gelassenen Kindes geschildert. Und genau an diesem Punkt setzt auch das Modell positiver Zuwendung und damit einhergehender Entwicklung ein. Es ist der Lehrer Habrecht, selbst ein Außenseiter in der Dorfgemeinschaft, der sich des Jungen annimmt. Habrecht versucht, Pavel Selbstachtung zu vermitteln und nimmt ihn vor den Anfeindungen und Verdächtigungen der Dörfler in Schutz. Als Pavel die Erlaubnis erhält, seine Schwester im Kloster zu besuchen, ist dies ein weiterer Einschnitt in seinem Leben. Er erkennt, dass außerhalb der dörflichen Zwänge ein Neubeginn leichter möglich wäre. Seine Bitte, als Knecht in der klösterlichen Landwirtschaft zu arbeiten, wird allerdings abgelehnt. In diesem entscheidenden siebten Kapitel zeigt EbnerEschenbach, die zur Schreibzeit unter dem kirchenkritischen Einfluss der ethischen Bewegung des Amerikaners William M. Salter stand, das abweisende Verhalten der Klosterfrauen in einem besonders negativen Licht. Wie das Schloss so ist auch das Kloster gegen die Außenwelt abgeschottet. Und so wie Ebner-Eschenbach die Haltung der Baronin kritisiert, so verurteilt sie auch die Herzenskälte und Selbstgerechtigkeit der Nonnen („unendlich fromm, unendlich teilnahmslos“, GK 197).

Mit dem elften Kapitel beginnt - formal abgetrennt - die zweite Hälfte der Erzählung: „Außerhalb des Dorfes, zu Füßen eines Abhangs, den vor Jahren der längst ausgerodete Bauernwald bedeckt hatte, befand sich eine verlassene Sandgrube." (GK 237) Pavel kauft dieses Fleckchen unwirtlichen Grund der Gemeinde viel zu teuer ab, um sich und seiner Mutter darauf ein Haus zu bauen. Die Arbeitsmöglichkeit außerhalb des Dorfes in der Sägemühle, in der Zuckerfabrik oder im Wald bringt ihm Geld und das wiederum verschafft ihm Eigentum. Für ihn bedeutet dies bereits einen sozialen Aufstieg, auch wenn die Anerkennung der Leute noch auf sich warten lässt. Die Wahl des Ortes für sein Haus symbolisiert 
auch seine Situation: frei stehend, aber auf einer Anhöhe und „weit draußen aus dem Dorf" (GK 253).

Nach dem Weggang des Lehrers Habrecht - seine Einrichtung bleibt gleichsam als materieller Teil seiner Hilfe bei Pavel zurück - muss sich sein Schützling alleine behaupten. Durch seinen Einsatz verhindert Pavel ein schweres Unglück mit dem neu angekauften Gemeindelokomobil; zum Dank dafür wird er von den Bauern dazu verurteilt, die Kosten für den dabei beschädigten Zaun zu tragen. Im Wirtshaus, wo traditionell die Hierarchien im Sozialgefüge der Gemeinde ausverhandelt werden, hält das einstige Gemeindekind den Bauern eine flammende Rede. Im Zentrum des Dorfes, in dem sich seine Gegner formieren, hält ihnen der selbstbewusst gewordene Pavel nun einen Spiegel vor. Als er aus der anschließenden Prügelei als Sieger hervorgeht und seinen Hauptgegner verschont, zollen ihm auch der Schmied Anton und der Förster ihre Anerkennung. Den Honoratioren schließt sich auch die Baronin an. Mit den räumlichen Schranken fällt damit auch die innere Distanz. Auf seiner Seite steht am Schluss aber auch - geläutert - der Hirt Virgil.

In seinem letzten Gespräch mit Milada im Kloster und bei seinem Zusammentreffen mit dem Lehrer Habrecht, der als Jünger der „Religion der Moral“ (GK 304) nach Amerika aufbricht, am Rande der Stadt erhält Pavel moralische Ratschläge und Lebensweisheiten für sein Wirken im „kleinen Kreise“ (GK 338). Marie von Ebner-Eschenbach verdeutlicht in dieser Rede des Lehrers auch ihre Sicht der sozialen Frage, wenn sie das Problem der Umverteilung anspricht:

In früheren Zeiten konnte einer ruhig vor seinem vollen Teller sitzen und sich's schmecken lassen, ohne sich darum zu kümmern, daß der Teller seines Nachbars leer war. Das geht jetzt nicht mehr, außer bei den geistig völlig Blinden. Allen übrigen wird der leere Teller des Nachbars den Appetit verderben, dem Braven aus Rechtsgefühl, dem Feigen aus Angst ... Darum sorge dafür, wenn du deinen Teller füllst, daß es in deiner Nachbarschaft so wenig leere als möglich gibt. Begreifst du? (GK, 307f.)

Pavels Weg führt zurück ins Dorf, wo er erstmals von Honoratioren wie Bauern respektiert wird. Zwar erhält er entscheidende Anstöße für seine positive Entwicklung immer wieder von außen, Ebner-Eschenbach gestattet ihrem Protagonisten trotz aller Schwierigkeiten aber keine Flucht aus seiner dörflichen Umgebung. Am Ende nimmt Pavel seine Mutter auf, die nach Verbüßung einer zehnjährigen Haftstrafe zurück ins Dorf kommt. Ein ungebrochenes Happy End ist das allerdings nicht. Dies hat schon Paul Heyse angemerkt, der an seine Kollegin Marie 
von Ebner-Eschenbach schreibt, er hätte dem Roman „zum Schluß ein wenig mehr Glück gegönnt. “38 Milada stirbt - sich selbst kasteiend - am stellvertretenden Leiden, in das sie von den Klosterfrauen gedrängt wird. Pavel wird trotz Besitzes allein bleiben, ohne Aussicht auf eine eigene Familie. Der Schluss mutet pathetisch und widersprüchlich an. ${ }^{39}$

Ebner-Eschenbachs Empathie für die Situation der Unterdrückten wurde von ihren Zeitgenossen spätestens seit ihren Dorf- und Schloßgeschichten anerkannt. Der österreichische Arbeiterführer Victor Adler, selbst Sohn einer wohlhabenden deutsch-jüdischen Kaufmannsfamilie aus Prag, schrieb am 27. Jänner 1890, zwei Wochen, bevor er auf vier Monate ins Gefängnis musste, an die adelige Schriftstellerin einen Brief mit der Bitte, Das Gemeindekind in der Arbeiter-Zeitung abdrucken zu dürfen. ${ }^{40}$ Dies ist bemerkenswert, war doch Ebner-Eschenbachs Verhältnis zur Sozialdemokratie distanziert. Sie setzte nicht auf das Kollektiv, sondern auf den Einzelnen, um die Gesellschaft zum Besseren $\mathrm{zu}$ verändern. Was mag Victor Adler für den Roman eingenommen haben? Das Landproletariat wird hier literaturwürdig, auch wenn dies bereits ansatzweise in den Dorfgeschichten Berthold Auerbachs oder in den Erzählungen und Romanen Jeremias Gotthelfs der Fall war. EbnerEschenbach geht aber noch einen Schritt weiter, indem sie Pavel zum "Träger der Geschichte“ und damit zum „Geschichtssubjekt" ${ }^{\text {“1 }}$ erhebt. Darüber hinaus setzt sie auf den Aspekt der Veränderung, der einen Weg aus der Misere zeigt. Diese Veränderung macht aber auch nicht vor dem Verhältnis zwischen Schloss und Dorf halt. Diese Beziehung ist brüchig geworden und wird nach dem Tod der Baronin endgültig zu Ende sein. Die Möglichkeit des Aufstiegs, die soziale Durchlässigkeit, der Appell, weiterzustreben und an sich weiterzuarbeiten, ist nicht nur an den Einzelnen gerichtet - und dies alles passte auch über alle ideologischen Grenzen hinweg gut in das Konzept der Arbeiterbewegung.

38. Paul Heyse an Marie von Ebner-Eschenbach, Brief v. 11. März 1887, in Mechthild Alkemade, Die Lebens- und Weltanschauung der Freifrau Marie von EbnerEschenbach. Mit 6 Tafelbeilagen und dem Briefwechsel Heyse und EbnerEschenbach. Graz und Würzburg, Wächter Verlag 1935, S. 303.

39. Philipp Hubmann spricht in seiner differenzierten Studie von einer „überkompensatorischen Verzichtserklärung" Pavels. Vgl. Hubmann, Biopolitik und Risikobewusstsein, 2016, S. 193.

40. Vgl. Rossbacher, Literatur und Liberalismus, 1992, S. 257-259 sowie 265f.

41. Pfeiffer, Marie von Ebner-Eschenbach, 2008, S. $132 \mathrm{f}$. 